EPJ Web of Conferences 19, 05012 (2012)

DOI: $10.1051 /$ epjconf/20121905012

(C) Owned by the authors, published by EDP Sciences, 2012

\title{
Solar-like pulsating stars as distance indicators: G-K giants in the CoRoT and Kepler fields
} \author{
and M. Valentini ${ }^{4}$ \\ 1 School of Physics and Astronomy, University of Birmingham, UK \\ 2 Institut d'Astrophysique et de Géophysique, Liège, Belgium \\ 3 Observatoire de la Côte d'Azur, Nice, France \\ 4 LESIA, Observatoire de Paris, France \\ 5 INAF, Osservatorio Astronomico di Padova
}

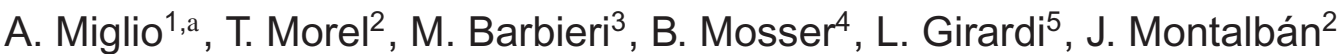

\begin{abstract}
The detection of radial and non-radial solar-like oscillations in thousands of G-K giants with CoRoT and Kepler is paving the road for detailed studies of stellar populations in the Galaxy. The available average seismic constraints allow a precise and largely model-independent determination of stellar radii (hence distances) and masses. We here briefly report on the distance determination of thousands of giants in the CoRoT and Kepler fields of view.
\end{abstract}

Thanks to the interpretation of solar-like oscillation spectra detected by CoRoT and Kepler [1-4], we can determine the mass and radius of thousands of stars belonging to the composite population of the Milky Way's disk. These innovative constraints allow precise estimates of distances and ages for giants, and will inform studies of galactic formation and evolution with observational constraints which were not available prior to asteroseismology (see e.g. [5-8] and references therein). We here briefly report on the distance determination of giants in the CoRoT and Kepler fields of view. A detailed description of the data, method, and results will be presented in a forthcoming paper.

As a first step we determine stellar radii by combining the available seismic parameters $v_{\max }$ and $\Delta v$ with effective temperatures $T_{\text {eff }}$. The latter are determined using 2MASS photometry and the colour- $T_{\text {eff }}$ calibrations by [9]. We then compute luminosities $L$ from $R$ and $T_{\text {eff }}$, and distances combining $L$ with de-reddened apparent 2MASS Ks magnitudes and bolometric corrections from [10]. Distance-dependent extinction from [11] is considered when determining the distance and $T_{\text {eff }}$. We estimate the uncertainty on the distances to be $\sim 10-15 \%$. This value can be further reduced when spectroscopic constraints will be available, and additional empirical tests of the scaling relations will be performed.

We apply this procedure to pulsating giants observed by CoRoT in several observational runs, and to giants in the public Kepler data [4]. The location in the Galaxy of 2500 CoRoT and 10000 Kepler targets is shown in Fig. 1 and Fig. 2. As presented in Fig. 3, the peak in the distribution of the absolute Ks magnitudes of giants (e.g. in CoRoT's LRc01 field) is in remarkable agreement with the absolute Ks magnitude of Hipparcos red-clump giants [12].

Studies are currently underway to combine distances with spectroscopic constraints, as well as with asteroseismic estimates of the mass (hence age) of these targets, leading to a detailed characterisation of populations of giants in different regions of the Milky Way.

\footnotetext{
a e-mail: a.miglio@bham.ac.uk
}

This is an Open Access article distributed under the terms of the Creative Commons Attribution-Noncommercial License 3.0, which permits unrestricted use, distribution, and reproduction in any noncommercial medium, provided the original work is properly cited. 
EPJ Web of Conferences

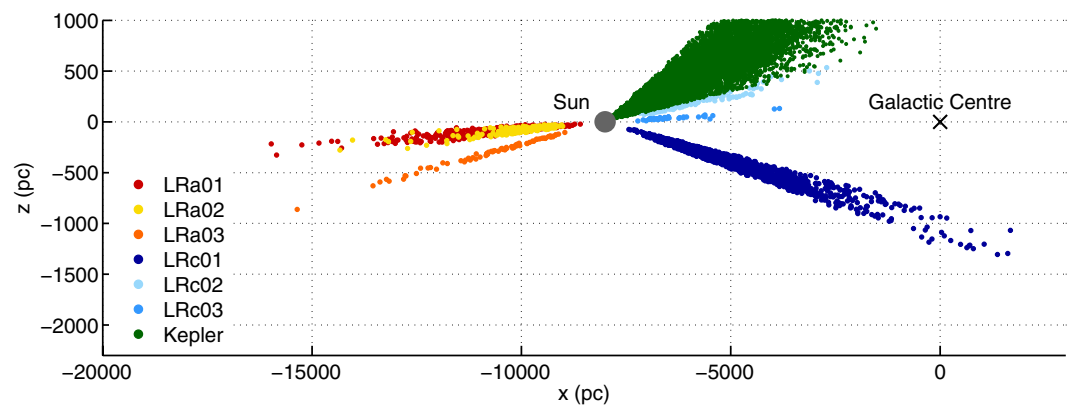

Figure 1. Solar-like oscillating G-K giants observed in several CoRoT fields of view and by Kepler: projection on the $x-z$ plane.

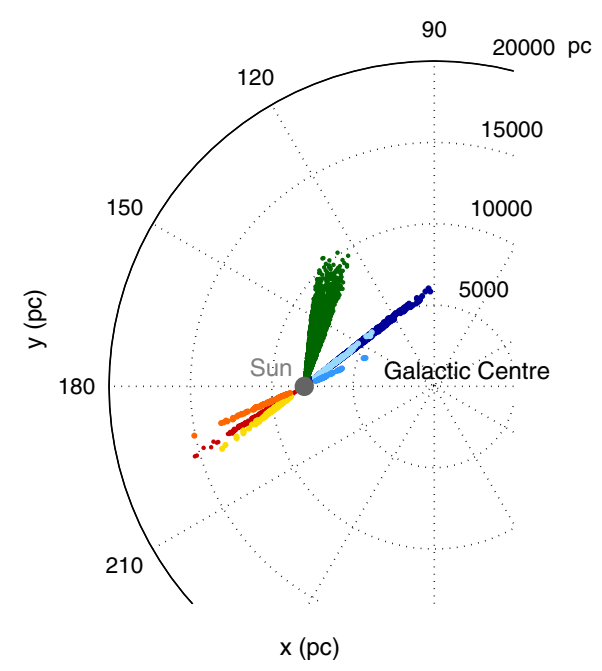

Figure 2. Projection on the Galactic plane of the stars shown in Fig. 1.

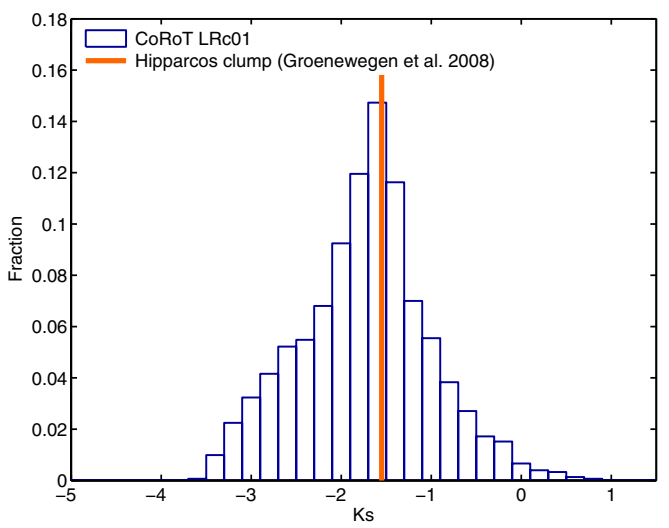

Figure 3. Distribution of the derived absolute Ks magnitude of solar-like pulsating giants in CoRoT LRc01. The vertical line shows the absolute magnitude of the Hipparcos red clump [12]. 
Assembling the Puzzle of the Milky Way

AM acknowledges financial support from the organisers and from the School of Physics and Astronomy, University of Birmingham.

\section{References}

[1] J. De Ridder et al., Nature 459, 398 (2009)

[2] B. Mosser et al., A\&A 517, A22 (2010)

[3] T.R. Bedding et al., ApJ 713, L176 (2010)

[4] S. Hekker et al., MNRAS 414, 2594 (2011)

[5] A. Miglio et al., A\&A 503, L21 (2009)

[6] A. Miglio, in Red Giants as Probes of the Structure and Evolution of the Milky Way, ApSS Proceedings, (2012), in press, http://arxiv.org/abs/1108.4555

[7] K. Freeman, in Red Giants as Probes of the Structure and Evolution of the Milky Way, ApSS Proceedings, (2012), in press, http://arxiv.org/abs/1108.5028

[8] C. Chiappini, in Red Giants as Probes of the Structure and Evolution of the Milky Way, ApSS Proceedings, (2012), in press

[9] A. Alonso, S. Arribas, C. Martínez-Roger, A\&AS 140, 261 (1999)

[10] L. Girardi, M.A.T. Groenewegen, E. Hatziminaoglou, L. da Costa, A\&A 436, 895 (2005)

[11] R. Drimmel, A. Cabrera-Lavers, M. López-Corredoira, A\&A 409, 205 (2003)

[12] M.A.T. Groenewegen, A\&A 488, 935 (2008) 